\title{
Including the Effects of Flexible Bearing Supports in Rotating Machinery
}

\author{
JOSÉ A. VÁZQUEZ*, LLOYD E. BARRETT ${ }^{\dagger}$ and RONALD D. FLACK ${ }^{\ddagger}$ \\ Department of Mechanical and Aerospace Engineering, School of Engineering and Applied Science, \\ University of Virginia, P.O. Box 400746, Charlottesville, Virginia 22904-4746, USA
}

(Received in final form 14 April 2000)

\begin{abstract}
Unbalance response and stability analyses of a flexible rotor on three lobe journal bearings on flexible supports are presented. The influence of the support structure was included in the analyses using polynomial transfer functions. These transfer functions were extracted from measured dynamic compliance data of the support structure, measured at the bearing locations. Numerical predictions using polynomial transfer functions and single mass supports are compared to the experimental data. Predictions using the transfer function representation of the support structure show a clear improvement over the predictions using single mass supports without over-complicating the problem. The predicted critical speeds are within $2.9 \%$ of the measured critical speeds. The predicted stability threshold agrees with the measured stability threshold within $1 \%$. The effects of cross talk between supports and cross coupling between horizontal and vertical directions are investigated. The cross talk between supports was found to have a strong influence in the results while the influence of cross coupling between the vertical and horizontal directions is negligible.
\end{abstract}

Keywords: Flexible supports; Rotating machinery; Experimental data; Stability; Unbalance response; Fluid film bearings

\section{INTRODUCTION}

The work presented in this paper is part of a larger study of the influence of flexible bearing supports on unbalance response and stability of rotating machinery (Vázquez, 1999). In this study, a flexible rotor is supported by two fluid film bearings on flexible supports. The dynamic behavior of the support structure is measured experimentally and included into the numerical analysis as polynomial transfer functions. One set of tilting pad bearings and two sets of three lobe bearings were used in conjunction with 15 support configurations. The rotor was tested for unbalance response and

\footnotetext{
*Corresponding author. Tel.: (804) 924-3292, Fax: (804) 982-2246, e-mail: jose@virginia.edu

†e-mail: leb@virginia.edu

†e-mail: rdf@virginia.edu
} 
stability on all support configurations and the three sets of fluid film bearings. It was not possible to drive the rotor unstable with the set of tilting pad bearings for the speed range of the experimental apparatus and only unbalance response was measured for this set of bearings.

This paper presents details for one of the support configurations used by Vázquez (1999) with one of the set of three lobe bearings. Vázquez summarized the results for this support configuration but no details were given. The analysis was also extended to include a study of the effects of cross coupling between vertical and horizontal directions and cross talk between supports.

The study started as an extension of the work by Lanes and Flack (1982). There, the authors carried out an experimental study of the effects of threelobe bearing geometry on rotor stability. The experimental apparatus used for those experiments was very similar to the one used here. The stability thresholds were calculated assuming rigid supports and compared with the experimental data. In all cases, the stability analysis under-predicted the measured stability threshold. It was proposed that the support flexibility was responsible for the difference. However, the analytical tools to include the support flexibility were not available at the time. Gash (1976) had proposed a method to include support flexibility into rotordynamic analyses. However, the method worked for unbalance response but was not suitable for stability analyses.

Hashish and Sankar (1984) developed the equations for the finite element approach for rotordynamic analysis, including bearing support flexibility. Nicholas and Barrett (1986) presented a method to include flexible support dynamics in rotor-dynamic analysis. They derived an expression of equivalent bearing coefficients when the bearing is mounted on flexible supports. The supports were modeled as a single mass system, represented by a single mass with springs and dampers in the horizontal and vertical directions.

Further development permitted the use of experimental data in unbalance response analyses (Barrett et al., 1986; Nicholas et al., 1986). This provided an improvement in the modeling of the supports but this new technique could not be used for stability analyses. Later, Rouch et al. (1989) presented some work where experimental frequency response functions (FRFs) were used to model the foundation of a rotor system for forced response analyses. The analysis was carried out using the finite element approach and the foundation characteristics were included frequency by frequency.

Redmond $(1995,1996)$ showed that under certain circumstances, substantial errors might occur when employing measured support data from impedance tests with the rotor installed. A method was included to subtract the dynamic effect of the rotor under these circumstances. The support models were represented by single mass systems.

Lees et al. (1998) and Feng and Hahn (1998) presented methods to obtain foundation dynamic characteristics from machine unbalance response.

Vázquez and Barrett $(1998,1999)$ presented a method to include the dynamic characteristics of bearing supports using transfer functions. These transfer functions could be calculated from measured frequency response functions, mode shape information or physical models of the support structure. The support transfer functions can be used in unbalance response and stability analyses. The experimental work presented by Vázquez (1999) complements the development of the analytical tools and shows that polynomial transfer functions can be used successfully to represent flexible supports of rotating machinery.

\section{EXPERIMENTAL APPARATUS}

The test apparatus has been fully described by Vázquez (1999) and Vázquez et al. (1999a, b). For the sake of brevity, only the most important details will be repeated. The test apparatus consists of a flexible shaft with three rigid disks equally spaced between two three-lobe bearings on anisotropic flexible bearing supports. The rotor has a maximum diameter of $25.4 \mathrm{~mm}$ and a minimum diameter of $19.0 \mathrm{~mm}$. The disks are rigidly 

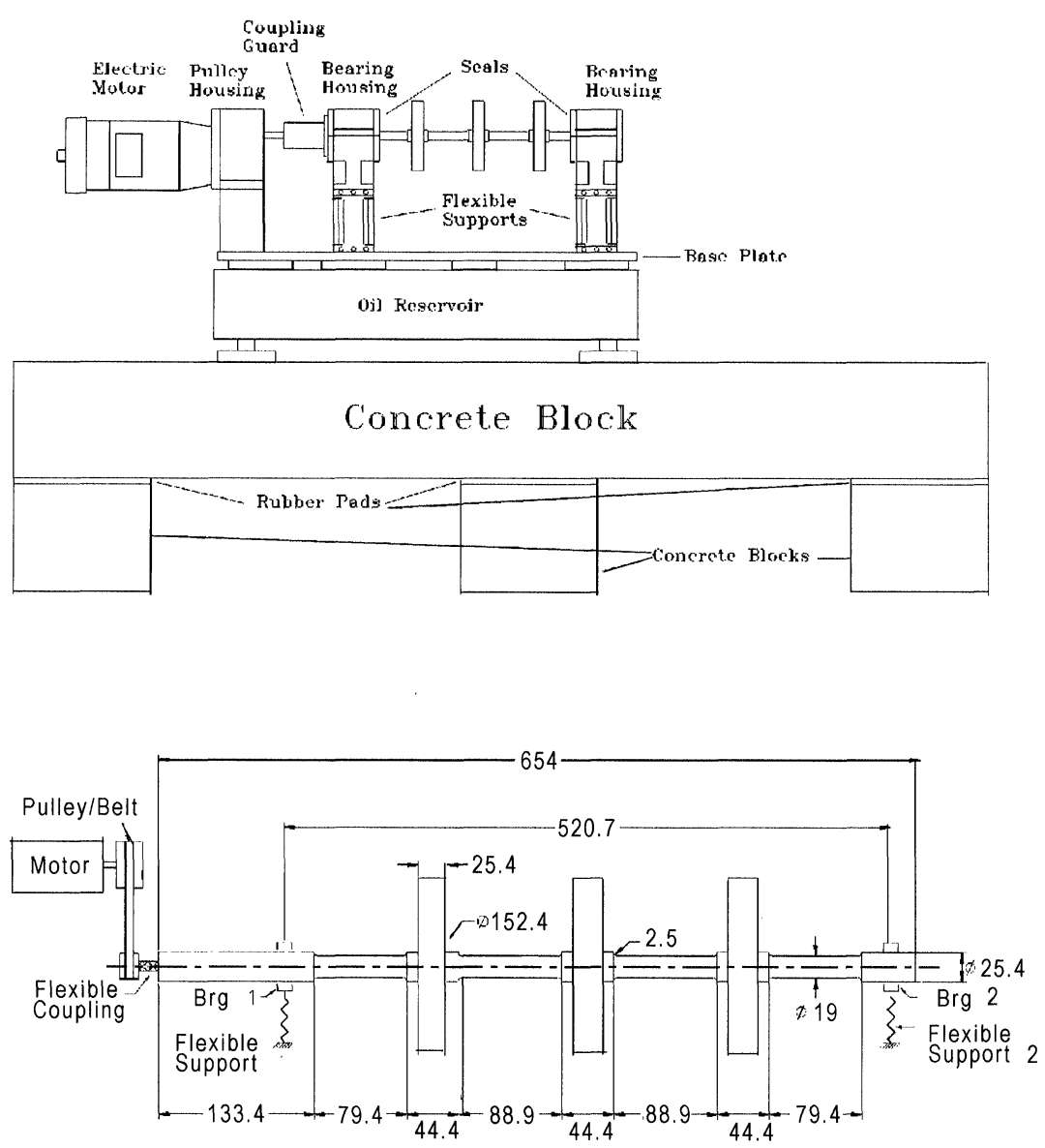

FIGURE 1 Experimental apparatus

attached to the rotor and have a diameter of $152.4 \mathrm{~mm}$. The total rotor length is $654.0 \mathrm{~mm}$ with a bearing span of $520.7 \mathrm{~mm}$. The total weight of the rotor is $12.64 \mathrm{~kg}$. The first and second critical speeds of the rotor are $2580 \mathrm{rpm}$ and $5100 \mathrm{rpm}$. A one-horsepower DC motor drives the rotor through a flat belt and flexible coupling. Any rotor speed up to $11000 \mathrm{rpm}$ can be obtained. The whole apparatus sits on top of a heated, oil-filled reservoir clamped to a $900 \mathrm{~kg}$ concrete block. Figure 1 shows the experimental apparatus.

The rotor is supported by two identical threelobe bearings. Table I shows the characteristics of the bearings while Figure 2 shows the calculated eight linear bearing stiffness and damping coefficients (Branagan, 1988). The bearings are supported by flexible elements that connect the rigid bearing housing to the oil reservoir. The flexible element design was based on a beam construction with stiffening plates connected at the

TABLE I Bearing characteristics

\begin{tabular}{lc}
\hline Journal diameter $(\mathrm{mm})$ & $25.400( \pm 0.0025)$ \\
Machined in pad radial clearance $(\mathrm{mm})$ & $0.1546( \pm 0.0017)$ \\
Bearing radial clearance $(\mathrm{mm})$ & \\
(clearance for a centered journal) & $0.0368( \pm 0.0017)$ \\
Bearing length $(\mathrm{mm})$ & $12.700( \pm 0.0025)$ \\
Bearing preload factor & $0.762( \pm 0.023)$ \\
Pivot offset factor & $0.484( \pm 0.012)$ \\
Lobe arc length $($ deg.) & $95( \pm 1)$ \\
Loading direction $($ deg. $)$ & $0.0( \pm 2.5)$ \\
Static load $(\mathrm{N})$ & $62.2( \pm 0.1)$ \\
Inlet pressure $(\mathrm{kPa})$ & $20.7( \pm 0.1)$ \\
Inlet temperature $\left({ }^{\circ} \mathrm{C}\right)$ & $48.3( \pm 0.5)$ \\
Oil viscosity $(\mathrm{Pa} \cdot \mathrm{s}) @ 40^{\circ} \mathrm{C}$ & 0.0262 \\
Oil viscosity $(\mathrm{Pa} \cdot \mathrm{s}) @ 55^{\circ} \mathrm{C}$ & 0.0159 \\
Oil density $\left(\mathrm{kg} / \mathrm{m}^{3}\right)$ & 908
\end{tabular}




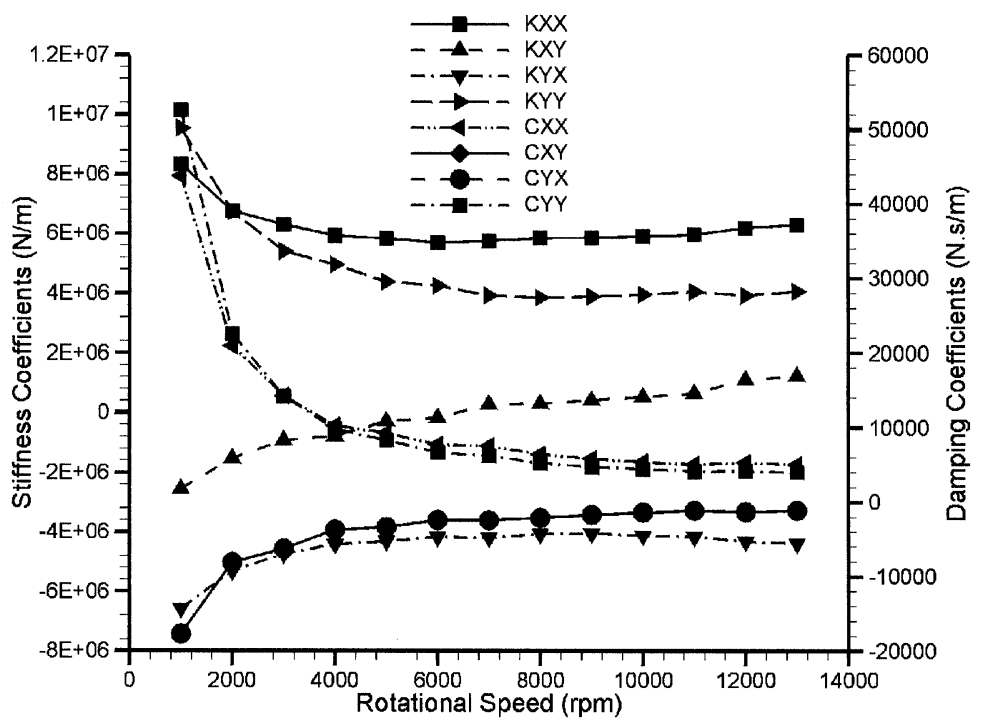

FIGURE 2 Bearing stiffness and damping coefficients.

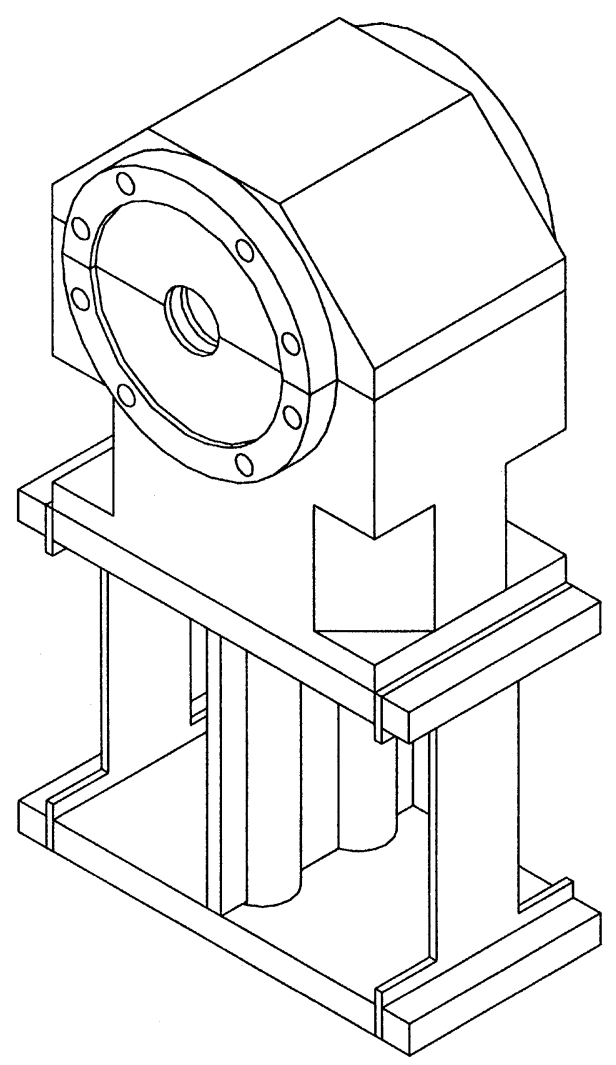

FIGURE 3 Bearing housing and flexible element. ends. It was constructed of aluminum. The oil drained from the bearing housing passed through drainpipes in the flexible element to the oil reservoir located under the assembly. Figure 3 shows a detail of the bearing housing with the flexible element. Figure 4 shows the design of the flexible support element with the stiffening plates. Changing the stiffening plates parametrically varies the horizontal stiffness of the support element. The stiffening plates used in this work were made of aluminum with a width of $63.5 \mathrm{~mm}$ and a thickness of $3.175 \mathrm{~mm}$.

\section{SUPPORTS FREQUENCY RESPONSE FUNCTIONS}

The dynamic characteristics of the supports were determined at the bearing locations. Frequency response functions (FRFs) of the support structure were obtained by exciting the support structure at the bearing housings and measuring the response. The rotor was removed during these tests. Direct, cross-coupled and cross talk FRFs were measured. These FRFs were later used to calculate polynomial transfer functions that would represent the 


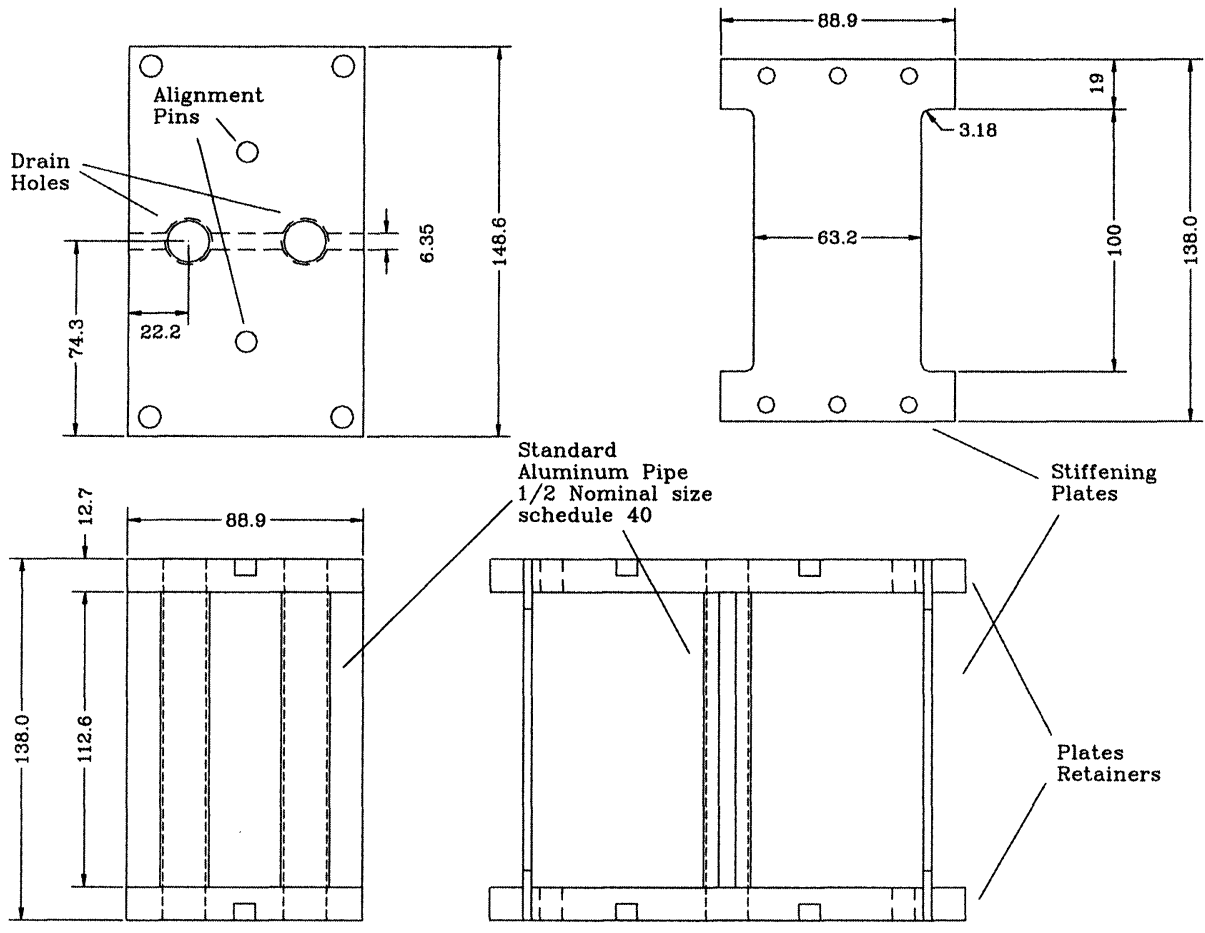

FIGURE 4 Design details of the flexible support elements.

support structure in the rotordynamic analyses. Polynomial transfer functions can be calculated using one of several system identification techniques available (Maia and Silva, 1997; Ljung, 1999).

A total of 16 FRFs were measured for the support structure. In matrix form they are organized as:

$\left\{\begin{array}{l}x_{1} \\ y_{1} \\ x_{2} \\ y_{2}\end{array}\right\}=\underbrace{\left[\begin{array}{ll:ll}d c_{x 1 x 1} & d c_{x 1 y 1} & d c_{x 1 x 2} & d c_{x 1 y 2} \\ d c_{y 1 x 1} & d c_{y 1 y} & d c_{y 1 x 2} & d c_{y 1 y 2} \\ \hdashline d c_{x 2 x 1} & d c_{x 2 y 1} & d c_{x 2 x 2} & d c_{x 2 y 2} \\ d c_{y 2 x 1} & d c_{y 2 y 1} & d c_{y 2 x 2} & d c_{y 2 y 2}\end{array}\right]}_{D C(\omega)}\left\{\begin{array}{l}f_{x 1} \\ f_{y 1} \\ f_{x 2} \\ f_{y 2}\end{array}\right\}$

where the numbers 1 and 2 refers to supports 1 and 2 respectively. The components of this matrix are measured one column at a time. That is, force is applied at one support in one direction and the responses for that column are measured simultaneously. Those FRFs that relate the displacement at one support with the force applied at the same support in the same direction are designated as direct frequency response functions. FRFs relating the response in one direction due to a force in the other direction are designated as cross coupling. Finally, those FRFs relating the response in one support to a force applied at the other support are designated cross talk.

The dynamic compliance matrix or FRF matrix is symmetric because the support structure is a passive system. Figures 5 through 7 show examples of the measured FRFs.

Figure 5 shows direct and cross talk FRFs in the horizontal direction. Notice that the direct FRFs are very similar. This indicates that supports 1 and 2 have a similar behavior in the horizontal direction. The cross talk FRFs are lined up on top of each other. This corroborates that the dynamic compliance matrix is indeed symmetric. 


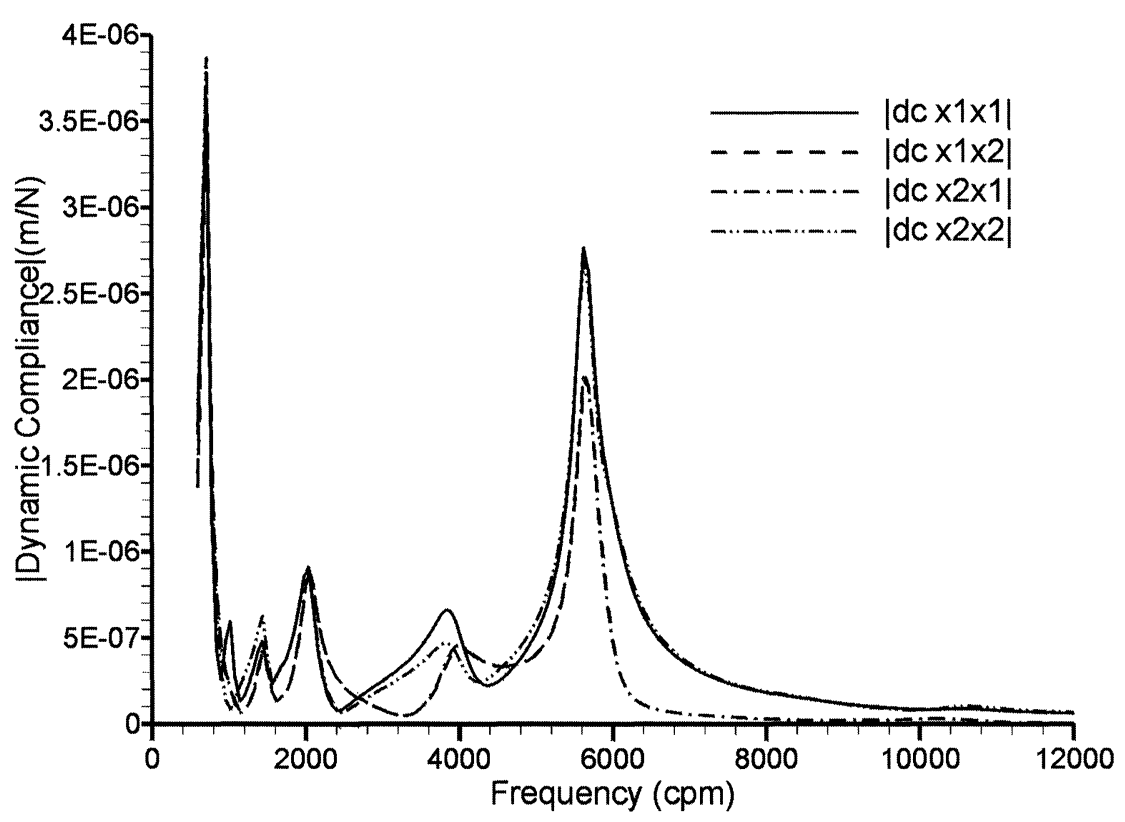

FIGURE 5 Measured direct and cross talk dynamic compliance in the horizontal direction.

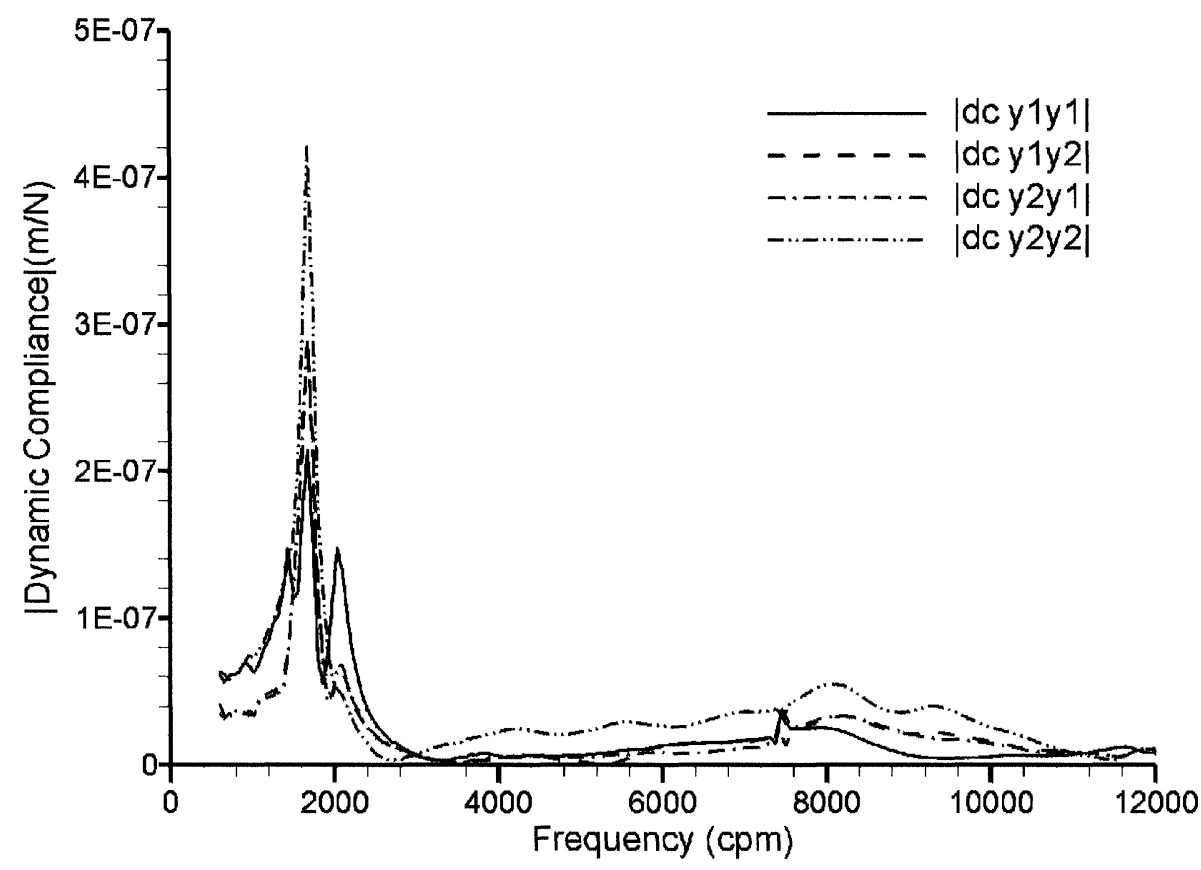

FIGURE 6 Measured direct and cross talk dynamic compliance in the vertical direction.

These cross-talk FRFs have the same order of magnitude as the direct FRFs, indicating a strong influence between supports.
Figure 6 shows the direct and cross talk FRFs in the vertical direction. Notice that the direct FRFs of each support are different but the same order of 
magnitude. This indicates that the supports are not as similar as expected, however, this does not affect the experiments since no assumption was made in the similarity of the supports. Again, the cross-talk FRFs are identical as expected for a passive system. Notice that the magnitude of the FRFs in the vertical direction is an order of magnitude smaller than the FRFs in the horizontal direction. This indicates that the supports are stiffer in the vertical direction, highlighting the anisotropy of the supports.

Figure 7 shows some cross-coupling FRFs. The magnitude of the FRFs is an order of magnitude smaller than the magnitude of the cross talk FRFs in the horizontal direction. From this, it is expected, and will be shown later, that the effect of the cross coupling in the support structure is not as strong as the cross talk between supports.

The frequency response functions were used to compute polynomial transfer functions using the method developed by Sanathanan and Koerner (1963) and later used by Gähler and Herzog (1994). The computed transfer functions form the transfer function matrix (TFM) of the support structure as:

$$
\left\{\begin{array}{l}
x_{1} \\
y_{1} \\
x_{2} \\
y_{2}
\end{array}\right\}=\underbrace{\left[\begin{array}{ll:ll}
g_{x 1 x 1} & g_{x 1 y 1} & g_{x 1 x 2} & g_{x 1 y 2} \\
g_{y 1 x 1} & g_{y 1 y 1} & g_{y 1 x 2} & g_{y 1 y 2} \\
\hdashline g_{x 2 x 1} & g_{x 2 y 1} & g_{x 2 x 2} & g_{x 2 y 2} \\
g_{y 2 x 1} & g_{y 2 y 1} & g_{y 2 x 2} & g_{y 2 y 2}
\end{array}\right]}_{G(s)}\left\{\begin{array}{c}
f_{x 1} \\
f_{y 1} \\
f_{x 2} \\
f_{y 2}
\end{array}\right\}
$$

The TFM is defined as a function of the complex frequency $s$ and is valid in the whole complex plane. The TFM has the same form as Eq. (1). However, the elements of the matrix in Eq. (1) are FRFs while each element of the TFM is a ratio of two polynomials, of the form:

$$
g_{i j}=\frac{a_{n} s^{n}+a_{n-1} s^{n-1}+\cdots+a_{1} s+a_{0}}{s^{m}+b_{m-1} s^{m-1}+\cdots+b_{1} s+b_{0}} \quad m>n
$$

In the case of these experiments, the polynomial transfer functions were of order 24 (i.e., $m=24$ ). This indicates that 12 modes were located in the range of operation. As an example, Table II shows

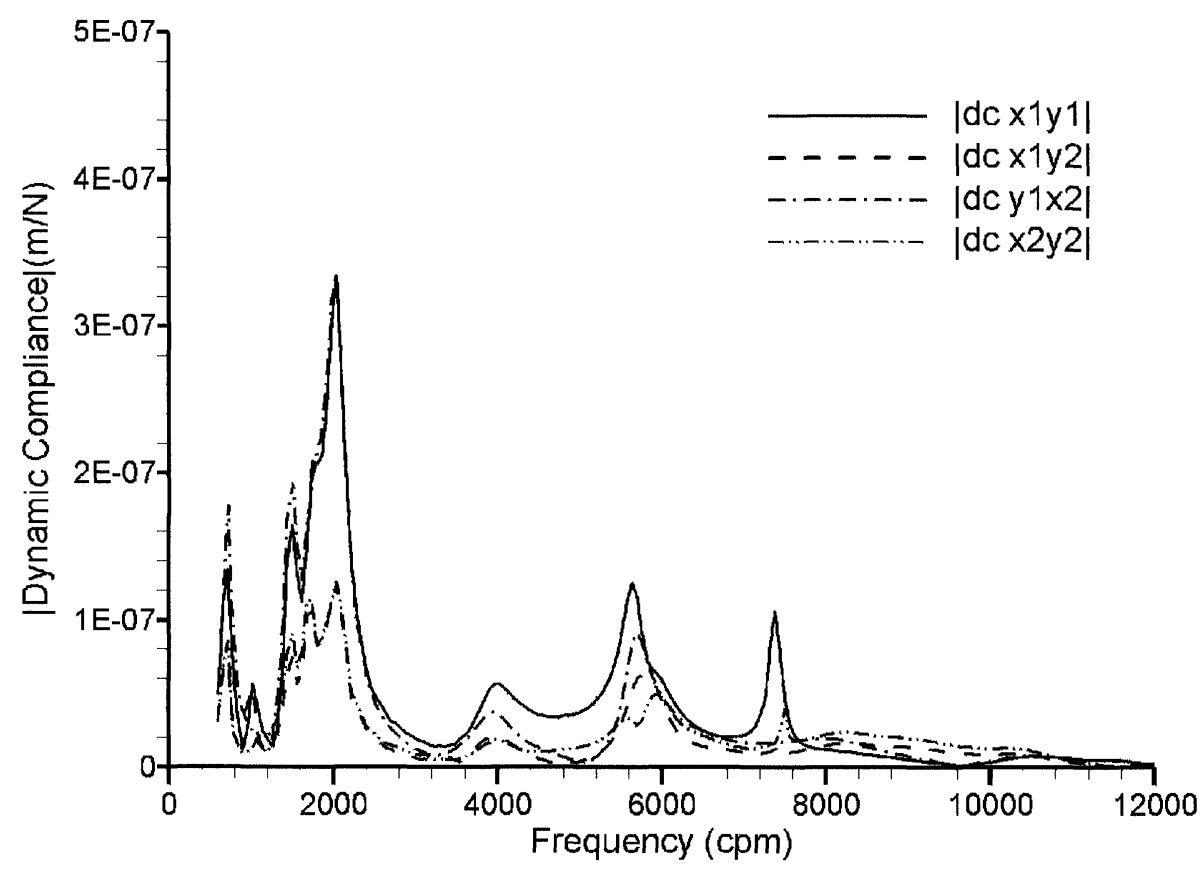

FIGURE 7 Measured cross coupling dynamic compliance. 
TABLE II Polynomial coefficients for $g_{x 1 x 1}$

\begin{tabular}{lcc}
\hline Power & Numerator & Denominator \\
\hline$s^{0}$ & $7.9978 E+50$ & $1.0703 E+57$ \\
$s^{1}$ & $1.0404 E+48$ & $1.8822 E+54$ \\
$s^{2}$ & $4.3296 E+47$ & $6.8793 E+53$ \\
$s^{3}$ & $6.4670 E+44$ & $1.1781 E+51$ \\
$s^{4}$ & $9.7153 E+43$ & $1.8147 E+50$ \\
$s^{5}$ & $1.5267 E+41$ & $3.0735 E+47$ \\
$s^{6}$ & $1.1547 E+40$ & $2.5336 E+46$ \\
$s^{7}$ & $1.7489 E+37$ & $4.1132 E+43$ \\
$s^{8}$ & $7.7874 E+35$ & $2.0145 E+42$ \\
$s^{9}$ & $1.0469 E+33$ & $2.9580 E+39$ \\
$s^{10}$ & $3.0112 E+31$ & $9.2519 E+37$ \\
$s^{11}$ & $3.3275 E+28$ & $1.1495 E+35$ \\
$s^{12}$ & $6.5359 E+26$ & $2.4278 E+33$ \\
$s^{13}$ & $5.4934 E+23$ & $2.3844 E+30$ \\
$s^{14}$ & $7.5753 E+21$ & $3.5245 E+28$ \\
$s^{15}$ & $4.4595 E+18$ & $2.5587 E+25$ \\
$s^{16}$ & $4.4121 E+16$ & $2.7141 E+23$ \\
$s^{17}$ & $1.6934 E+13$ & $1.3984 E+20$ \\
$s^{18}$ & $1.2666 E+11$ & $1.1190 E+18$ \\
$s^{19}$ & $2.8349 E+07$ & $3.9151 E+14$ \\
$s^{20}$ & $1.6561 E+05$ & $2.4477 E+12$ \\
$s^{21}$ & $1.5627 E+01$ & $5.2719 E+08$ \\
$s^{22}$ & $7.2548 E-02$ & $2.6053 E+06$ \\
$s^{23}$ & $-1.0472 E-06$ & $1.0000 E+00$ \\
$s^{24}$ & $0.0000 E+00$ & \\
\hline & & \\
\hline & & \\
& & \\
& &
\end{tabular}

the polynomial coefficients for the transfer function $g_{x 1 \times 1}$.

An equivalent support stiffness matrix is calculated from the TFM as:

$$
\left[K_{\text {sup }}(s)\right]=[G(s)]^{-1}
$$

This equivalent support stiffness matrix is frequency dependent and can be used to represent the support structure for unbalance response and stability analysis.

\section{SINGLE MASS SUPPORTS}

Single mass supports are the most common kind of models used to represent flexible supports. They consist of a mass with springs and dampers in the vertical and horizontal directions. The equivalent support stiffness matrix for this kind of supports is written as:

$$
\left[K_{\text {sup }}(s)\right]=s^{2}\left[M_{s}\right]+s\left[C_{s}\right]+\left[K_{s}\right]
$$

where $\left[M_{s}\right],\left[C_{s}\right]$ and $\left[K_{s}\right]$ are the support structure mass, damping and stiffness matrices. For the case of two flexible bearing supports (as is the case here) the equivalent support stiffness matrix is written as:

$$
\begin{aligned}
{\left[K_{\text {sup }}(s)\right]=s^{2} } & {\left[\begin{array}{cccc}
m_{1} & 0 & 0 & 0 \\
0 & m_{1} & 0 & 0 \\
0 & 0 & m_{2} & 0 \\
0 & 0 & 0 & m_{2}
\end{array}\right] } \\
+ & =\left[\begin{array}{cccc}
C_{x 1 x 1} & 0 & 0 & 0 \\
0 & C_{y 1 y 1} & 0 & 0 \\
0 & 0 & C_{x 2 x 2} & 0 \\
0 & 0 & 0 & C_{y 2 y 2}
\end{array}\right] \\
+ & {\left[\begin{array}{cccc}
K_{x 1 x 1} & 0 & 0 & 0 \\
0 & K_{y 1 y 1} & 0 & 0 \\
0 & 0 & K_{x 2 x 2} & 0 \\
0 & 0 & 0 & K_{y 2 y 2}
\end{array}\right] }
\end{aligned}
$$

Equation (6) does not include cross coupling between the vertical and horizontal directions or cross talk between supports. This is the standard practice for support structure modeling with single mass supports because these coefficients are, at best, difficult to estimate.

The single mass support models used in this work the properties:
$m_{1}=m_{2}$
$12.7 \mathrm{~kg}$
$K_{x 1 \times 1}$
$1.3410^{6} \mathrm{~N} / \mathrm{m}$
$K_{y 1 y 1}$
$3.7810^{7} \mathrm{~N} / \mathrm{m}$
$C_{x 1 x 1}=C_{y 1 y 1}$
$0.00 \mathrm{~N}-\mathrm{s} / \mathrm{m}$
$K_{x 2 \times 2}$
$1.9510^{6} \mathrm{~N} / \mathrm{m}$
$K_{y 2 y 2}$
$3.2110^{7} \mathrm{~N} / \mathrm{m}$
$C_{x 2 x 2}=\mathrm{C}_{y 2 y 2}$
$0.00 \mathrm{~N}-\mathrm{s} / \mathrm{m}$

The mass of the supports was determined by weighing the rigid bearing housings and bearings. The stiffness of the supports was calculated by extrapolation of the FRF to zero frequency. This operation can be done directly on the FRF plots. An alternative way is to calculate the transfer function matrix, derived in the previous section, at 
zero frequency or:

$$
\left[K_{s}\right]=[G(0)]^{-1}
$$

This calculation of the support stiffness is approximate and depends on the validity of the extrapolation. The assumption is that the transfer function of the supports is well behaved outside the range of the experimental data.

The value for the support damping was assumed to be zero. This is also a common practice since the estimation of the damping is very difficult.

The support stiffness to bearing stiffness ratio for support 1 is between 0.16 and 0.23 for the horizontal direction and between 3.96 and 9.80 for the vertical direction. For support 2, the support stiffness to bearing stiffness ratio is between 0.23 and 0.34 in the horizontal direction and between 3.36 and 8.33 for the vertical direction.

\section{SUPPORT STIFFNESS IN ROTORDYNAMIC ANALYSES}

The previous two sections developed the calculation method of the equivalent support stiffness matrix. This matrix has the same form for the single mass supports and the transfer function representation. This section will show how to include this matrix into rotordynamic analyses. We will assume that the reader is familiar with rotordynamic analysis in general and the generation of the dynamic matrices for a rotor supported on fluid film bearings (Ehrich, 1992). The equations of motion of a rotor on rigidly supported fluid film bearings can be described as:

$$
\begin{gathered}
s^{2}\left[M_{r}\right]\left\{u_{r}\right\}+s[G]\left\{u_{r}\right\}+\left[K_{r}\right]\left\{u_{r}\right\} \\
+\left[K_{b}(s)\right]\left\{u_{r}\right\}=\{f\}
\end{gathered}
$$

where:

$\left[M_{r}\right],\left[K_{r}\right]$ are the mass and stiffness matrices of the rotor

$[G]$ is the matrix of the gyroscopic effect
$\left[K_{b}(s)\right]$ is the complex bearing coefficient matrix. It includes the stiffness and damping coefficients of all the bearings in the system. One can group the rotor matrices into a single frequency dependent matrix:

$$
[\operatorname{Rotor}(s)]\left\{u_{r}\right\}+\left[K_{b}(s)\right]\left\{u_{r}\right\}=\{f\}
$$

Equation (9) is a general representation of the rotor system on fluid film bearings. Other effects can be added to this equation without loss of generality. Flexible bearing supports can be added to Eq. (9) as an additional level. The equations of motion of the rotor on flexible supports are:

$$
\begin{aligned}
& {\left[\begin{array}{c:c}
{[\operatorname{Rotor}(s)]+\left[K_{b}(s)\right]_{r r}} & -\left[K_{b}(s)\right]_{r s} \\
\hdashline-\left[K_{b}(s)\right]_{s r} & {\left[K_{b}(s)\right]_{s s}+\left[K_{\text {sup }}(s)\right]}
\end{array}\right]\left\{\begin{array}{c}
u_{r} \\
u_{s}
\end{array}\right\}} \\
& =\left\{\begin{array}{l}
f \\
\underline{0}
\end{array}\right\}
\end{aligned}
$$

Equation (10) represents the general equation of motion for rotordynamic analysis. The sub-indices in the bearing matrices indicate that the rows and columns are adjusted to the proper size. For forced response analyses:

$$
s=i \omega,\{f\}=\{\hat{f}\} e^{i \omega t}
$$

for this type of analysis, the frequency of vibration is known and the goal is to find the displacements $\left\{u_{r} \mid u_{s}\right\}^{T}$. Unbalance response is a special case of forced response where the excitation forces vary as a function of the rotational speed.

For stability analyses:

$$
s=p \pm i \omega_{d},\{f\}=\{\underline{0}\}
$$

For this kind of analysis the goal is to calculate the complex frequencies $s$. This analysis is usually transformed into an eigenvalue problem where the vibration frequencies $s$ are the eigenvalues and the displacement vectors $\left\{u_{r} \mid u_{s}\right\}^{T}$ are the eigenvectors. The sign of the real part of the eigenvalues determines the stability of the system. If the real part of all eigenvalues is negative, the system is stable. If the real part of any of the eigenvalues is 
positive or zero, the system is unstable. Because the bearing properties and the gyroscopic effects are speed dependent, stability must be calculated at different rotor speeds. In this framework, instability threshold is defined as the rotor speed where one or more of the eigenvalues have the real part equal to zero.

\section{UNBALANCE RESPONSE}

The unbalance response of the rotor was measured by applying known unbalances to the rotor and measuring the response during rotor run-up from $1000 \mathrm{rpm}$ to $7000 \mathrm{rpm}$. Data was recorded every $10 \mathrm{rpm}$. The average acceleration of the rotor was $3.5 \mathrm{rad} / \mathrm{s}^{2}$. For each unbalance distribution, the unbalance response was measured twice. The second time, the unbalance weights were located $180^{\circ}$ from the original location. The unbalance response of the rotor due to the applied unbalance distribution is obtained by subtracting the response of the second run from the response of the first run and dividing the result by 2 . This operation eliminates the effects of mechanical and electrical run-out, shaft bow and residual unbalance.

Figure 8 shows the measured unbalance response near the center disk for an unbalance of $8.92 \mathrm{~g}-\mathrm{mm}$ located at $225^{\circ}$ (lead) on the center disk. This unbalance was designed to excite the first critical speed of the rotor. This figure shows three peak responses. The peaks at $1900 \mathrm{rpm}$ and $4000 \mathrm{rpm}$ correspond to structural resonances. The second response peak, at $2580 \mathrm{rpm}$, is the first critical speed of the rotor.

This figure shows unbalance response predictions using all the transfer functions, neglecting the cross-talk terms, neglecting the cross-coupling terms and using single mass supports. The prediction of the unbalance response using the single mass supports does not agree with the experimental data. This model predicts two peak responses at the locations of the support resonances and does not show a peak at the first critical speed of the rotor.

The analysis using the polynomial representation of the support structure shows good agreement with the experimental data. The predictions are comparable in all cases. Using all the transfer functions predicts the first critical speed at $2540 \mathrm{rpm}$ or $1.6 \%$ below the measured critical speed. The prediction assuming independent supports (no cross talk between supports) shows the first critical speed at $2510 \mathrm{rpm}$ or $2.7 \%$ below the measured critical speed. Finally, the analysis ignoring the cross-coupling effects in the supports shows a critical speed at $2560 \mathrm{rpm}$ or $0.7 \%$ below the measured critical speed.

Figure 9 shows the measured and predicted unbalance response near the right disk for an unbalance of $22.86 \mathrm{~g}-\mathrm{mm}$ at $30^{\circ}$ (lead) on the left disk and an unbalance of $22.86 \mathrm{~g}-\mathrm{mm}$ at $210^{\circ}$ (lead) on the right disk. This unbalance distribution was designed to excite the second critical speed. This figure shows the second critical speed at $5100 \mathrm{rpm}$. Coupling with the first mode of vibration of the rotor causes the small response at $2580 \mathrm{rpm}$.

The predicted response using the single mass supports fails to accurately predict the second critical speed of the rotor, the second critical speed was predicted at $10030 \mathrm{rpm}$. In the range of interest, this model predicts a response at $2910 \mathrm{rpm}$.

The analysis using all the transfer functions and ignoring the cross-coupling terms show the same response, indicating that the cross coupling between the horizontal and vertical directions does not have a large effect in this case. The predicted second critical is $5250 \mathrm{rpm}$ or $2.9 \%$ above the measured critical speed. The predicted magnitude of the response is $3.0 \%$ below the measured amplitude at the critical speed.

The analysis with the supports considered independent of each other (no cross talk between supports) does not agree with the experimental data. The predicted second critical speed is 


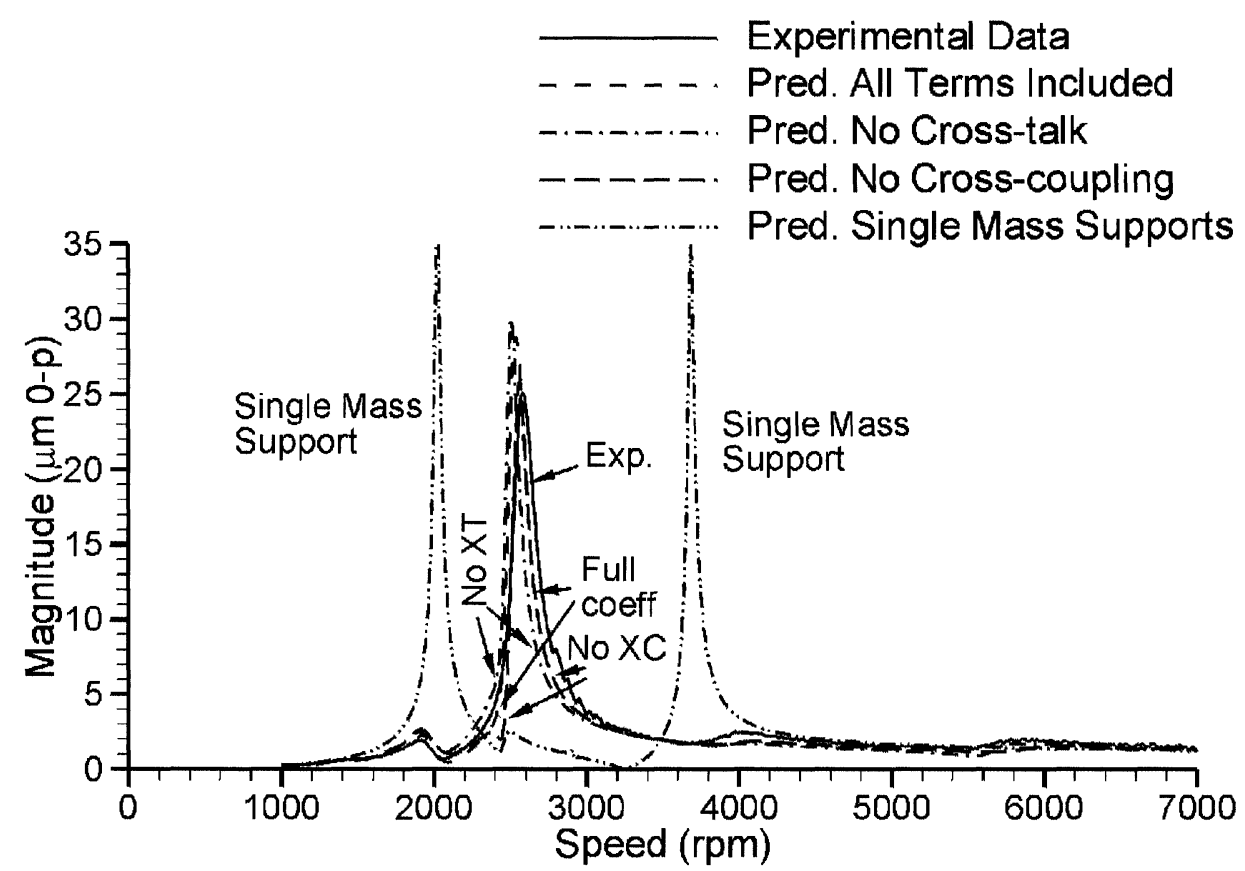

FIGURE 8 Measured and predicted unbalance responses near the middle disk in the horizontal direction. Unbalance distribution 1 .

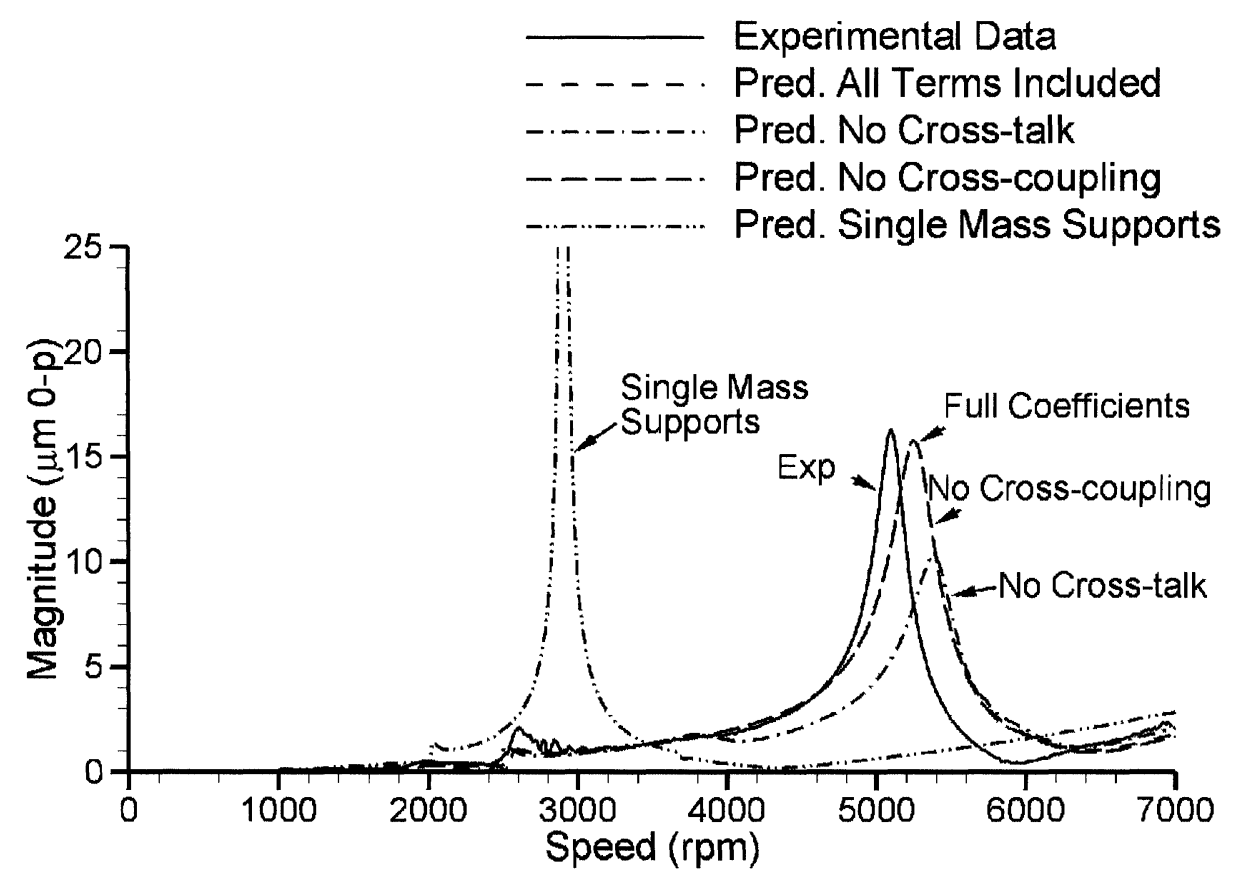

FIGURE 9 Measured and predicted unbalance responses near the right disk in the horizontal direction. Unbalance distribution 2 . 
$5390 \mathrm{rpm}$ or $5.7 \%$ above the measured critical speed while the predicted magnitude at the second critical speed is $37.4 \%$ smaller than the measured magnitude at the critical speed. This shows that the cross talk between supports has a significant effect in this experimental setup.

\section{STABILITY}

For stability analysis, the rotor was accelerated until the stability threshold was reached. A spectral map was created from $3000 \mathrm{rpm}$ until the stability threshold of $9960 \mathrm{rpm}( \pm 120 \mathrm{rpm})$ with a speed resolution of $120 \mathrm{rpm}$ and a frequency resolution of $2 \mathrm{~Hz}(120 \mathrm{cpm})$. Figure 10 shows the spectral map of the displacement signal at the center disk during run-up.

The stability threshold was defined as the speed where sub-synchronous vibrations were self-sustained and grew in time. The definition of the stability threshold is important in this case because the system is lightly damped. Under this condition, sub-synchronous vibrations may be present but the overall vibration of the system does not increase in time. Figure 10 shows the synchronous vibration $(1 X)$ and the vibration at two times the running speed $(2 X)$. The instability threshold is clearly marked in the figure.

Figure 11 shows the stability map calculated for the rotor bearing system using different support models. The instability threshold was defined as the speed at which the logarithmic decrement of one or more of the eigenvalues of the system was zero. The logarithmic decrement is defined as:

$$
\delta=-2 \pi \frac{p}{\omega_{d}}
$$

where $p$ and $\omega_{d}$ are the real and imaginary parts of the eigenvalue (defined in Eq. (12)).

The stability threshold measured from Figure 10 is included in Figure 11 for comparison. The prediction using the single mass model of the supports does not agree with the measured stability threshold. Using polynomial transfer functions for the support structure improves the prediction.

The stability analysis using all the transfer functions predicts the stability threshold at

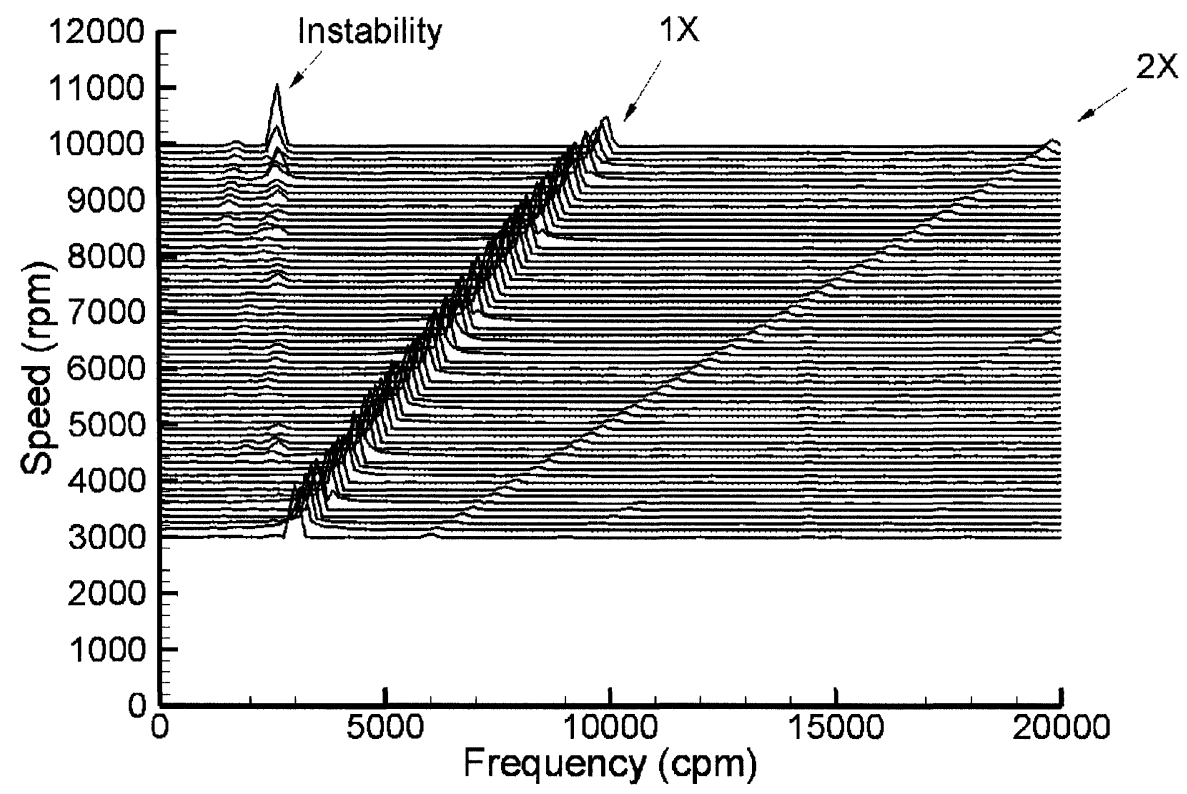

FIGURE 10 Spectral map of the vibration displacement at the middle disk. 


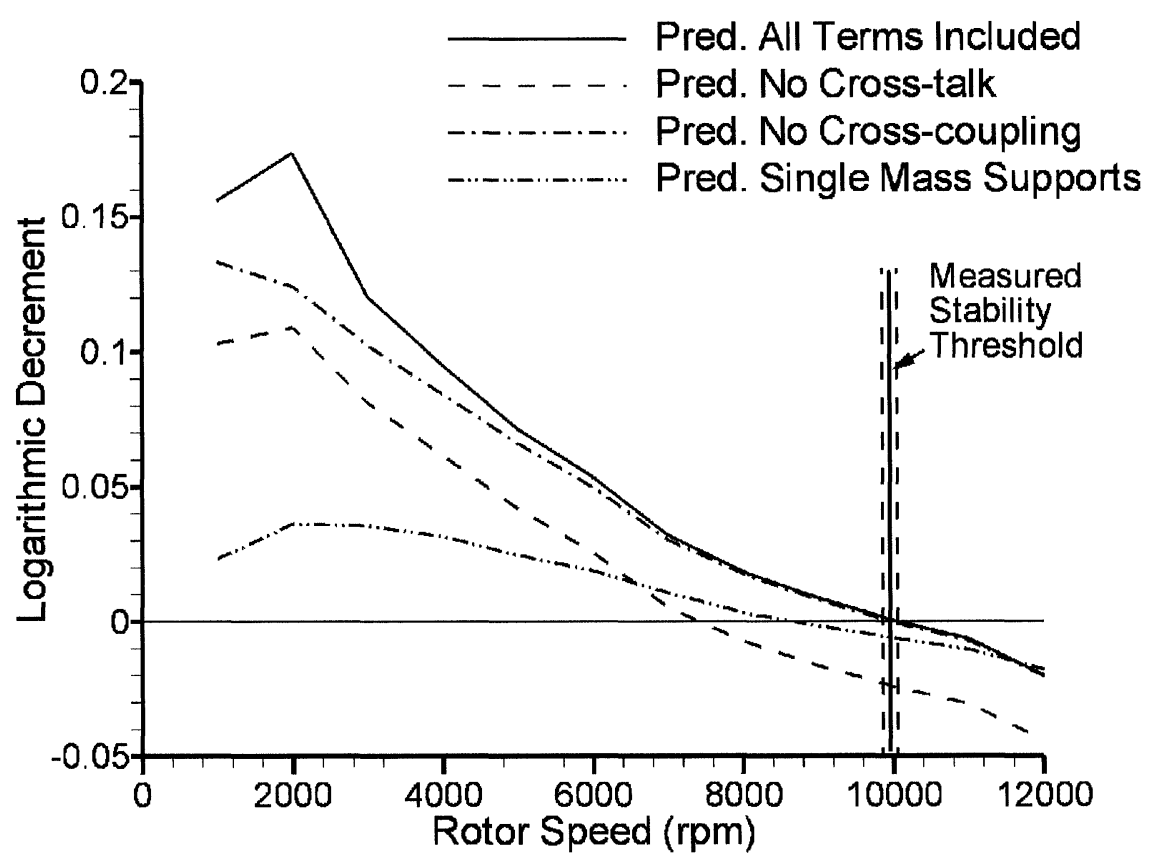

FIGURE 11 Stability maps using different flexible support models.

$10050 \mathrm{rpm}(0.9 \%$ above the measured stability threshold and within the experimental uncertainty). The stability analysis ignoring the cross coupling in the support predicts the stability threshold at $9946 \mathrm{rpm}(0.01 \%$ below the measured stability threshold and within the experimental uncertainty). This again indicates that the support cross coupling does not have a large effect in this system.

The stability analysis assuming independent supports (no cross talk) predicts the stability threshold at $7410 \mathrm{rpm}$. This prediction does not agree with the measured stability threshold, indicating that the cross talk between supports has a significant effect in this system.

\section{SUMMARY AND CONCLUSIONS}

An experimental apparatus with a flexible rotor supported by two identical three-lobe bearings on flexible supports was used in this research. Frequency response functions of the support structure were measured at the bearing locations with the rotor removed. Polynomial transfer functions were calculated from these frequency response functions and used to represent the support structure in rotordynamic analyses.

Numerical analyses using polynomial transfer functions agreed with the experimental data. The first critical speed was calculated within $1.6 \%$ of the measured critical speed. The second critical speed was calculated within $2.9 \%$ of the measured speed. The stability threshold of the rotor was calculated within $0.9 \%$ of the measured stability threshold and within the experimental uncertainty.

Cross coupling between the $X$ and $Y$ directions does not have a large influence in the calculated results. On the other hand, cross talk has an important effect in the prediction of the second critical speed and stability threshold.

For comparison purposes, the supports were modeled using single mass supports. The models were created using the mass of the bearing and bearing housing and the estimated static stiffness 
of the supports. These models failed to capture the support effects for unbalance response and stability.

\section{Acknowledgements}

This research was sponsored by the Rotating Machinery and Controls Laboratories (ROMAC) at the University of Virginia.

\section{References}

Barrett, L. E., Nicholas, J. C. and Dhar, D. (1986) The dynamic analysis of rotor-bearing systems using experimental bearing support compliance data, Proceedings of the Fourth International Modal Analysis Conference, Union College, Schenectady, New York, pp. 1531-1535.

Branagan, L. A. (1988) Thermal analysis of fixed and tilting pad journal bearings including cross-film viscosity variations and deformations, Ph.D. Dissertation, University of Virginia, Charlottesville, VA.

Erich, F. F. (1992) Handbook of Rotordynamics, John Wiley \& Sons, Inc.

Feng, M. S. and Hahn, E. J., On the identification of a flexibly supported rigid foundation with unknown location of the principal axes of inertia, Proceedings of ISROMAC-7, the 7th International Symposium on Transport Phenomena and Dynamics of Rotating Machinery, Honolulu, Hawaii, February, 1998 , pp. $705-714$.

Gähler, C. and Herzog, R., Identification of magnetic bearing systems, Proceedings of the Fourth International Symposium on Magnetic Bearings, ETH Zurich, August, 1994, pp. $293-298$

Gash, R. (1976) Vibration of larger turbo-rotors in fluid-film bearings on an elastic foundation, Journal of Sound and Vibration, 47(1), 53-73.

Hashish, E. and Sankar, T. S., Finite element and modal analyses under stochastic loading conditions, Journal of Vibration, Acoustics, Stress, and Reliability in Design, Vol. 106, January, 1984, pp. 81-89.

Lanes, R. F. and Flack, R. D., Effects of three-lobe bearing geometries on flexible rotor stability, ASLE Transactions, 25(3), July, 1982, 377-385.

Lees, A. W., Friswell, M. I., Smart, M. G. and Prells, U., The identification of foundation vibration parameters from running machine data, Proceedings of ISROMAC-7, the 7th
International Symposium on Transport Phenomena and Dynamics of Rotating Machinery, Honolulu, Hawaii, February, 1998, pp. 715-724.

Ljung, L. (1999) System Identification, Theory for the user. Second Edition, Prentice Hall, Inc.

Maia, N. M. M. and Silva, J. M. M. (1997) Theoretical and Experimental Modal Analysis, Research Studies Press LTD.

Nicholas, J. C. and Barrett, L. E. (1986) The effect of bearing support flexibility on critical speed prediction, $A S L E$ Transactions, 29(3), 329-338.

Nicholas, J. C., Whalen, J. K. and Franklin, S. D. (1986) Improving critical speed calculations using flexible bearing support FRF compliance data, Proceedings of the 15th Turbomachinery Symposium, Texas A\&M University, College Station, Texas.

Redmond, I. (1995) Practical Rotordynamics Modeling Using Combined Measured and Theoretical Data, Proceedings of the 13th International Modal Analysis Conference, Nashville.

Redmond, I., Rotordynamic Modelling Utilizing Dynamic Support Data Obtained From Field Impact Tests, Paper C500/055/96, Proceedings of Sixth International Conference on Vibrations in Rotating Machinery, Oxford, Sept., 1996.

Rouch, K. E., McMains, T. H. and Stephenson, R. W. (1989) Modeling of rotor-foundation systems using frequencyresponse functions in a finite element approach, 1989 ASME Design Technical Conference 12th Biennial Conference on Mechanical Vibration and Noise, Montreal, Canada, pp. $157-166$.

Sanathanan, C. K. and Koerner, J., Transfer function synthesis as a ratio of two complex polynomials, IEEE Transactions on Automatic Control, January, 1963, pp. 56-58.

Vázquez, J. A. and Barrett, L. E. (1998) Representing flexible supports by polynomial transfer functions, ASME paper 98-GT-27.

Vázquez, J. A., Using transfer functions to model flexible supports and casings of rotating machinery, Ph.D. Dissertation, University of Virginia, Charlottesville, VA, January, 1999.

Vázquez, J. A. and Barrett, L. E., Transfer function representation of flexible supports and casings of rotating machinery, Proceedings of the 17th International Modal Analysis Conference, 8-11, February, 1999, Kissimmee, Florida.

Vázquez, J. A., Barrett, L. E. and Flack, R. D., A flexible rotor on flexible bearing supports. Part I: Stability, Paper DETC99/VIB-8285, Proceedings of the 1999 Vibration Conference, 12-16, September, 1999a, Las Vegas, Nevada.

Vázquez, J. A., Barrett, L. E. and Flack, R. D., A flexible rotor on flexible bearing supports. Part II: Unbalance Response, Paper DETC99/VIB-8286, Proceedings of the 1999 Vibration Conference, 12-16, September, 1999b, Las Vegas, Nevada. 

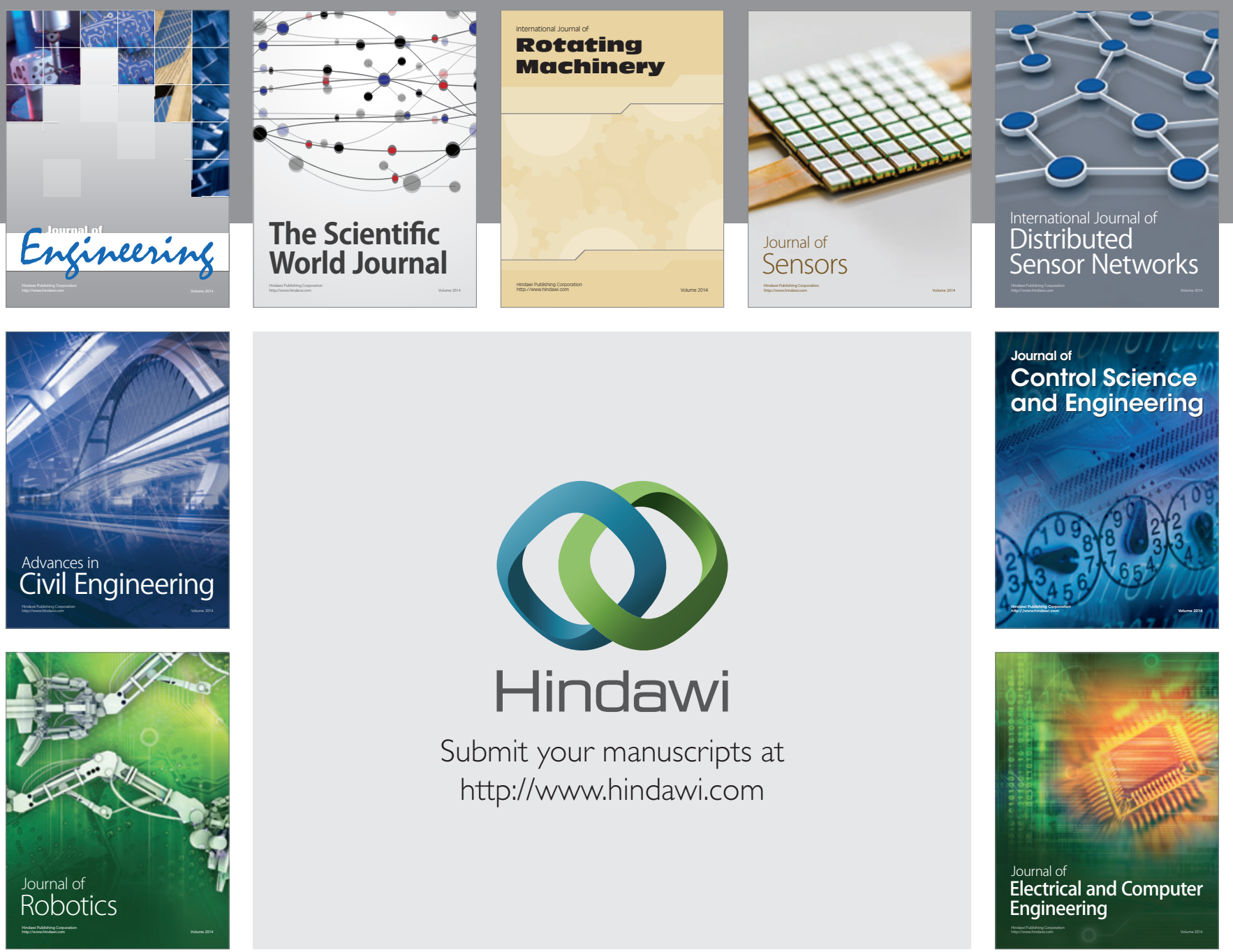

Submit your manuscripts at

http://www.hindawi.com
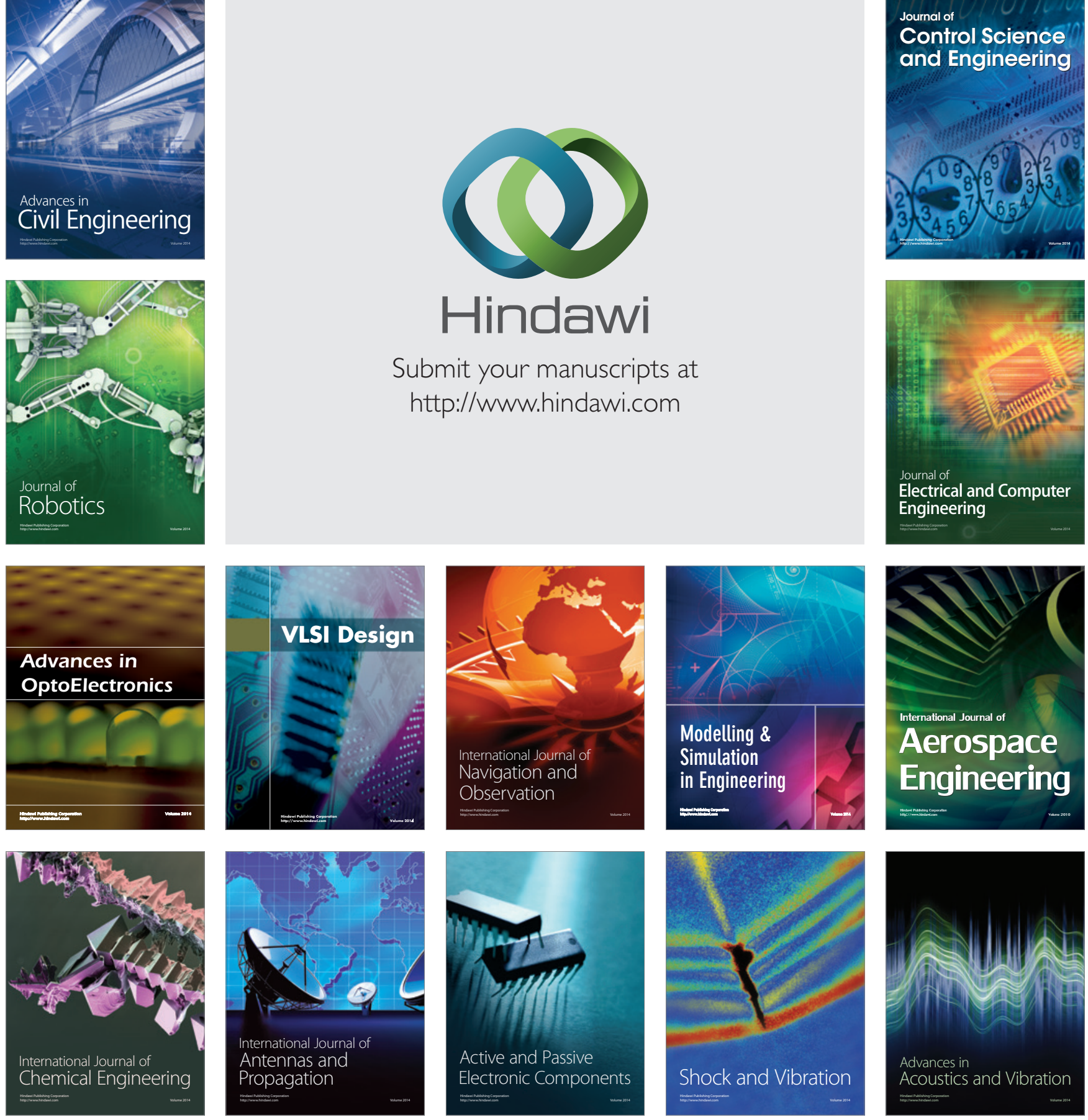